\title{
ESTUDO COMPARATIVO DA ABUNDÂNCIA DA FAMILIA CHRYSOPIDAE DA FLORESTA NACIONAL DE PACOTUBA-ES, CAPTURADOS NAS DISTINTAS FASES LUNARES
}

\author{
ATAIDE, Julielson Oliveira ${ }^{1}$ \\ SILVA FILHO, Gilson ${ }^{2}$
}

RESUMO: O presente avaliou a abundância da família de Chrysopidae na Floresta Nacional de Pacotuba em distintas fases lunares, por meio de armadilhas atrativas, com uma coleta em cada fase da lua do mês, no período de setembro de 2012 a Agosto de 2013. Foram avaliados os seguintes parâmetros: dossel denso, de menor densidade, riqueza, abundância, dominância, índices de diversidade e uniformidade de Shannon (H' e E'), dominância de Berger-Parker (BP) e Pielou: J'. As estimativas de riqueza foram feitas através dos procedimentos: Jackknife1 e Margalef. Foram coletados 1416 exemplares pertencentes ao gênero Leucochrysa, 12 espécies sendo 887 no dossel denso e 529 no de menor densidade, tendo as fases não luminosas (Minguante e Nova) maior abundância e ambas as áreas. Os valores analisadas para período total foram: Shannon $(0,4462)$, Margalef $(1,517)$, Berger-Parker $(0,9066)$ e Pielou J (0.1796). Apresentou maior quantidade de exemplares no dossel denso e nas fases lunares não luminosas, já os fatores de precipitação, velocidade do vento e temperatura se mostram significantes. A riqueza de Chrysopidae foi relativamente baixa quando comparado com outros trabalhos realizados em ecossistemas florestais com o mesmo grupo taxonômico.

Palavras-Chave: Biodiversidade. Lua Minguante. Lua Nova. Lua Crescente. Lua Cheia.

\section{COMPARATIVE STUDY OF THE CHRYSOPIDAE FAMILY ABUNDANCE OF FLORESTA NACIONAL DE PACOTUBA-ES, CAPTURED IN THE DIFFERENT LUNAR PHASES}

SUMMARY: The present evaluated the abundance of the Chrysopidae family in the Floresta Nacional de Pacotuba in different lunar phases, by means of attractive traps, with a collection in each moon phase of the month, from September 2012 to August 2013. Following parameters: dense, lower density canopy, richness, abundance, dominance, Shannon diversity and uniformity indices (H 'and $\left.\mathrm{E}^{\prime}\right)$, Berger-Parker dominance (BP) and Pielou: J '. Wealth estimates were made using the following procedures: Jackknife1 and Margalef. A total of 1416 specimens belonging to the genus Leucochrysa were collected, 12 species being 887 in the dense canopy and 529 in the lowest density. The values analyzed for the total period were: Shannon (0.4462), Margalef (1.517), Berger-Parker (0.9066) and Pielou $\mathbf{J}$ (0.1796). It presented larger number of specimens in the dense canopy and in the non-luminous lunar phases, but the precipitation factors, wind speed and temperature are significant. Chrysopidae richness was relatively low when compared to other works carried out in forest ecosystems with the same taxonomic group.

Keywords: Biodiversity. Waning Moon. New Moon. Crescent moon. Full moon.

\section{INTRODUÇÃO}

A família Chrysopidae tem uma ampla distribuição na região neotropical, constituindo um dos grupos de predadores mais utilizados em programas de controle biológico de pragas em nível mundial (TAUBER et al., 2000), predando presas diversas, tais como pulgões, cochonilhas, ácaros e muitos outros pequenos artrópodes com tegumento facilmente perfurável (CARVALHO; SOUZA, 2000).

\footnotetext{
${ }^{1}$ Universidade Federal do Espirito Santo

${ }^{2}$ Centro Universitário São Camilo
} 
Esta família merece maior atenção quanto a sua sistemática, pois até 2012 eram poucos os trabalhos sistemáticos que avaliavam a diversidade de espécies neste grupo taxonômico, resumindo-se ao desenvolvido por Costa et al. (2010) avaliando as taxocenoses do grupo em distintas formação em Minas Gerais. Também o trabalho desenvolvido por Multani (2008) avaliando a riqueza e atividade de espécies de Chrysopidae em agroecossistema e Silva-Filho (2011) em ecossistema florestal no Bioma Mata Atlântica, ambos no estado do Rio de Janeiro. Os poucos trabalhos desenvolvidos tinham como base a descrição taxonômica proposta, principalmente por Brooks; Barnard (1990) e Freitas; Penny (2001), muitas com erros de descrição taxonômica. No espírito Santo o único trabalho que tinha sido desenvolvido avaliando a riqueza de Chrysopidae no Espírito Santo foi o de Pontes (2012) na reserva Biológica Sooretama. Desde 2012 Silva-Filho vêm desenvolvendo levantamentos sistematizados em distintos ecossistemas (florestal, restinga, mangue) no bioma Mata Atlântica. Mesmo com o aumento das pesquisas com Chrysopidae tenham aumentado no sudeste, ainda existem muitas espécies a serem descobertas ainda, conforme evidenciado em todos os trabalhos com esse grupo no sudeste, visto que todos os aspectos do controle biológico dependem de uma base sistemática sólida. Visando essas realidades pesquisadores vêm fazendo levantamentos para que possibilite a comunicação e o acesso à literatura científica e oferece uma perspectiva filogenética comparativa que é essencial para o entendimento das relações entre as pragas e seus inimigos naturais (TAUBER et al., 2000; TAUBER et al., 2001 ; DÍAZ-ARANDA et al., 2001).

Os crisopídeos são predominantes tanto em diversidade como abundância nos ecossistemas florestais, mas também tem se adaptado a agroecossistemas como pomares, seringais e eucaliptais (ALBUQUERQUE; TAUBER, 2001). Multani (2008), estudando a atividade da fauna de Chrysopidae em sistemas agrícolas, observou que os gêneros Leucochrysa e Ceraeochrysa têm suas atividades de vôo similares como previsto por (DUELLI et al. ,2002). Multani (2008) evidenciou que as espécies dos dois gêneros, Leucochrysa e Ceraeochrysa, iniciaram sua atividade de vôo logo após o pôr-do-sol. Multani (2008) observou picos de atividades uma hora depois de iniciarem suas atividades. Este autor avaliou a atividade de Chrysopidae mediante a utilização de armadilhas atrativas, iscadas com solução de melado de cana de açúcar.

Silva-Filho (2011), em sua pesquisa de doutorado, realizou as coletas de crisopídeos florestais, com armadilha atrativa proposta por multani, geralmente na última semana de cada mês dos anos de 2007 e 2008. Essa padronização pode proporcionar coletas de adultos sempre sobre a influência da mesma fase lunar ou de fazes muito próximas. Isto pode favorecer a captura deste táxon, com distintos padrões de riqueza e abundância de espécies, as quais tenham sofrido influências do efeito das fases da lua.

Os efeitos que incidem na captura com armadilhas são principalmente os relacionados à biologia do inseto alvo, tipo de guilda trófica e alimentar, e variáveis ambientais como temperatura, precipitação, velocidade do vento, umidade e fases da lua. Os efeitos da influência das variáveis sazonais na abundância crisopídeos foram evidenciados por Multani (2008) e Silva-Filho (2011). Grande parte dos trabalhos verificaram apenas as influências das variáveis ambientais na abundância e riqueza de insetos. Quanto às fases da lua, muitos verificam a influência na captura de insetos utilizando armadilhas luminosas como nos trabalhos de Sant'ana; Lozovei (2001) e Delfina; Teston (2010) analisaram a influência do ciclo lunar na coleta de insetos. O efeito da lua na captura e atividade de insetos também foi verificado por Willians; Singh (1951), utilizando armadilha de sucção, onde foi constatada diferença entre as fases "luminosas" (crescente e cheia) e as "não luminosas" (minguante e nova). O objetivo do presente trabalho foi avaliar a influência das fases da lua na abundância e na riqueza da família Chrysopidae.

Nucleus,v.16,n.2,out.2019 


\section{MATERIAL E MÉTODO}

\section{Descrição da área de estudo}

A pesquisa foi realizada na região da Floresta Nacional de Pacotuba, gerenciada pelo ICMBIO, localizada no município de Cachoeiro de Itapemirim - Espírito Santo. Possui área de aproximadamente 450 hectares, situada a $20^{\circ} 45^{\prime} \mathrm{S}$ e $41^{\circ} 17^{\prime} \mathrm{W}$ e com altitude igual a $100 \mathrm{~m}$. O clima da região é do tipo Aw (Köppen), clima tropical caracterizado por um verão quente e chuvoso e um inverno seco e frio.

\section{Coleta de dados}

Foi avaliada a variação das fases lunar, no calendário de 2012 e 2013, para evidenciar a faixa média de variação mensal do período de ocorrência da fase lunar. Assim foi possível correlacionar a fase da lua com o período de instalação das armadilhas atrativas para crisopídeos. As coletas foram realizadas de setembro de 2012 a setembro de 2013, seguindo o calendário lunar de 2012 e 2013, mediante a utilização de armadilhas atrativas, iscada com solução de cana-de-açúcar diluída em água a 5\%. As armadilhas atrativas foram confeccionadas por garrafas PET (volume de dois litros). A garrafa possuía duas aberturas opostas de 3 x $3 \mathrm{~cm}$, a $10 \mathrm{~cm}$ da base conforme metodologia utilizada por Silva-Filho (2011). A solução de melado era preparada e depositada nas garrafas $(200 \mathrm{ml})$ dois dias antes da fase lunar, para permitir a fermentação prévia do mesmo, tornando-a mais atrativa na durante a fase.

O melado permanecia na armadilha por três dias e era retirado um dia após a fase lunar. Foram utilizadas 40 armadilhas distribuídas em uma área aberta, dossel com baixa densidade, e em uma área com denso dossel. Os insetos capturados eram transportados em potes exclusivos ao Centro Universitário São Camilo - ES para identificação das espécies. Foram avaliadas a riqueza e abundância em cada fase lunar.

\section{Análises dos dados}

Os crisopídeos foram avaliados quanto à sua frequência de ocorrência $(\mathrm{FO})$ e abundância relativa (AR). Este tipo de avaliação já foi realizado para inventários de outros grupos de insetos (SILVEIRA NETO et al., 1976; BUSCHINI, 2000, SILVA-FILHO,2011). A FO é igual ao número de amostras com a espécie i dividido pelo número total de amostras e multiplicado por 100 . Se $\mathrm{FO} \geq 50 \%$, a espécie é considerada como muito frequente ( $\mathrm{mf}$ ); se $\mathrm{FO}<50 \%$ e $\geq 25 \%$, a espécie é considerada como frequente (f), e se FO $<25 \%$, a espécie é considerada como pouco frequente (pf). A AR foi calculada como a abundância da espécie i dividida pela abundância total e multiplicado por 100 . Quando AR $\geq 5 \%$, a espécie é considerada muito abundante (ma); se $\mathrm{AR}<5 \%$ e $\geq 2,5 \%$, a espécie é considerada abundante (a), e quando $\mathrm{AR}<2,5 \%$, a espécie é considerada pouco abundante (pa).

A diferença da abundância entre as fases luminosas e não luminosas e entre o dossel menos denso e mais denso foram avaliadas pelo teste do quí-quadro sob 5\% de significância com auxílio do programa Bioestat 5.0 (AYRES et al., 2007)

A diversidade, mensurada mediante a utilização do índice de Shannon, $H^{\prime}=-\sum$ pi ln pi, onde: pi $=$ proporção de indivíduos da espécie i representados na amostra, $\ln =$ logaritmo neperiano com o auxílio do programa Past (HAMMER et al., 2003).

A riqueza foi obtida pelo índice de Margalef, $D_{\operatorname{mag}}=(S-1) / \ln N$, onde: $S=$ número de espécies e $N$ é o número total de indivíduos. Tanto a diversidade de Shannon quanto a riqueza de Margalef foram calculadas com auxílio do programa PAST (HAMMER et al., 2003). A riqueza provável de cada área 
encontrada foi calculada com o auxílio do programa EstimateS 8.2.0 para Windows (COLWELL, 2009), pelo cálculo do estimador Jackknife1: $\mathrm{S}_{\mathrm{jack} 1}=\mathrm{S}_{\mathrm{obs}}+\mathrm{Q} 1 \mathrm{x}(\mathrm{m}-1 / \mathrm{m})$, onde $\mathrm{S}_{\mathrm{obs}}=$ riqueza observada, $\mathrm{Q} 1=$ número de espécies presentes em somente 1 agrupamento e $\mathrm{m}=$ número de agrupamentos que contém a $\mathrm{i}^{\text {ésima }}$ espécie de um agrupamento. O resultado para Jackknifel estima a riqueza total da área, somando a riqueza observada a um parâmetro calculado a partir do número de espécies raras e do número de amostras. Este estimador foi calculado com auxílio do programa EstimateS 8.2 para Windows (COLWELL, 2009). Permite estimar o número máximo de espécies que poderiam ser capturadas em uma área se o esforço amostral fosse aumentado (SANTOS, 2003).

A dominância da comunidade foi obtida pelo índice de Berger-Parker, $d=N m a x / N$, onde: Nmax é o número de indivíduos da espécie mais abundante e $\mathrm{N}$ o número total de indivíduos amostrados na área (MAGURRAM, 2004). A uniformidade da distribuição de abundância entre as espécies foi calculada segundo a fórmula de Pielou: J' = H'/Hmax, onde: H' é o índice de Shannon e Hmax é o logaritmo neperiano (ln) do número total de espécies na amostra (Magurran, 2004). Tanto a dominância quanto a uniformidade da comunidade foram calculadas com o programa Past. Estes índices possuem uma vantagem que, para uma mesma comunidade, eles tendem a assumir um valor constante em relação a aumentos no esforço amostral, e são frequentemente utilizados na literatura (MELO, 2008).

Foram aplicadas e comparadas curvas de rarefação para a riqueza de espécies com 1.000 aleatorizações para as áreas estudadas conforme Magurran (2004). Essa análise foi realizada com o auxílio do programa PAST (HAMMER et al., 2003). As curvas de rarefação permitiram padronizar e comparar os dados de riqueza obtidos em cada área, com a utilização dos dois métodos de captura (COLWEELL; CODDINGTON, 1994, SANTOS, 2003, MAGURRAN, 2004).

Para verificar a influência da temperatura, umidade e precipitação no aumento ou redução da abundância e riqueza de crisopídeos, foram realizadas análises de regressão linear para cada área de amostragem em relação à riqueza e abundância com auxílio do programa STATISTICA 7.0 para Windows (STATSOFT; INC., 2004), com significância de 5\% na análise de variância.

\section{RESULTADO E DISCUSSÃO}

Foram capturados 1416 crisopídeos adultos pertencentes a 12 espécies da tribo Leucochrysini. Das espécies capturadas Leucochrysa (Leucochrysa) varia com 1275 adultos foi a mais abundante, enquanto que de Leucochrysa (Nodita) digitiformis e Leucochrysa (Nodita) sp5 foram menos abundantes, com apenas um indivíduo (Tabela 1). Neste trabalho o número de espécies capturadas foi relativamente pequeno, quando comparado aos resultados dos trabalhos de Silva-Filho (2011) nas duas áreas, pequenos remanescentes de Mata Atlântica, com área menor que 50 ha, adjacentes aos fragmentos florestais maiores que 100 ha e a mata contínua. Este autor coletou o mínimo de 16 espécies numa área que tinha 25 ha. Pontes (2012), estudando a fauna deste táxon no Espírito Santo, evidenciou que as áreas da BR e da trilha do quirinão, com fitofisionomia mais esparsa, foram os que apresentaram menor constituição específica, mesmo assim superior ao evidenciado nesse trabalho. 
Tabela 1. Número de indivíduos por espécie da família Chrysopidae na Floresta Nacional de Pacotuba, capturados com armadilha atrativa contendo solução de melado de cana-de-açúcar, durante os anos de 2012 e 2013.

\begin{tabular}{|c|c|c|c|c|c|c|c|c|c|c|c|c|c|}
\hline \multirow{2}{*}{ ESPÉCIES } & \multicolumn{4}{|c|}{2012} & \multicolumn{8}{|c|}{2013} & \multirow{2}{*}{ TOTAL } \\
\hline & set & out & nov & dez & jan & fev & mar & abri & mai & jun & Jul & ago & \\
\hline L. (L.) varia & 179 & 131 & 62 & 405 & 350 & 28 & 8 & 2 & 61 & 13 & 14 & 28 & 1281 \\
\hline L. (L.) boxi & 0 & 0 & 1 & 0 & 0 & 0 & 0 & 0 & 1 & 0 & 0 & 0 & 2 \\
\hline L. $(N$.$) digitiformis$ & 1 & 0 & 0 & 0 & 0 & 0 & 0 & 0 & 0 & 0 & 0 & 0 & 1 \\
\hline L. (N.) duarte & 5 & 0 & 0 & 1 & 3 & 0 & 1 & 0 & 0 & 0 & 0 & 0 & 10 \\
\hline L. (N.) cruentata & 6 & 2 & 10 & 3 & 27 & 0 & 0 & 0 & 17 & 5 & 4 & 4 & 78 \\
\hline L. $(N.) \mathrm{sp} 1$ & 1 & 0 & 1 & 3 & 0 & 0 & 0 & 0 & 1 & 0 & 0 & 1 & 7 \\
\hline L. (N.) $\mathrm{sp} 2$ & 1 & 0 & 0 & 0 & 0 & 0 & 0 & 0 & 0 & 0 & 0 & 1 & 2 \\
\hline L. (L.) $\mathrm{sp} 3$ & 10 & 3 & 1 & 3 & 0 & 0 & 0 & 0 & 0 & 0 & 1 & 0 & 18 \\
\hline L. $(N.) \mathrm{sp} 4$ & 1 & 1 & 1 & 0 & 0 & 0 & 0 & 0 & 0 & 0 & 0 & 0 & 3 \\
\hline L. (N) $\mathrm{sp} 5$ & 0 & 0 & 0 & 1 & 0 & 0 & 0 & 0 & 0 & 0 & 0 & 0 & 1 \\
\hline L. (N.) sp6 & 0 & 0 & 0 & 0 & 7 & 0 & 0 & 0 & 1 & 0 & 0 & 0 & 8 \\
\hline G. nigriceps & 0 & 0 & 2 & 0 & 0 & 0 & 0 & 0 & 0 & 0 & 0 & 0 & 2 \\
\hline Total & 204 & 137 & 78 & 416 & 387 & 28 & 9 & 2 & 81 & 18 & 19 & 34 & 1416 \\
\hline
\end{tabular}

Os resultados evidenciaram que das espécies capturadas, apenas duas foram consideradas como comuns, L. $(L)$ varia e L.(N.) cruentata, 4 espécies raras e as demais acessórias. Essa distribuição de dados também foi evidenciadas nos trabalhos de Silva-Filho (2011) onde os autores registraram algumas espécies apenas como comuns e raras e mais da metade capturada como espécies acessórias. Isso pode estar evidenciando a dominância de nichos por algumas espécies, similar ao modelo Random Fraction proposto por Tokechi (1999), onde a abundância de uma espécie é muito superior que as demais (Tabela 2).

Tabela 2: Representação das Categorias de Abundância de Chrysopidae capturados na Floresta Nacional de Pacotuba de 2012 a 2013 com armadilha atrativa iscada com solução de melado de cana-de-açúcar.

\begin{tabular}{|c|c|c|c|}
\hline & $\overline{\mathrm{FO}^{\mathrm{a}}}$ & $\mathrm{AR}^{\mathrm{b}}$ & $\overline{\mathrm{CA}^{\mathrm{c}}}$ \\
\hline L.(L.) varia & $\mathrm{mf}$ & $\mathrm{ma}$ & $\mathrm{C}$ \\
\hline L.(L.) boxi & $\mathrm{pf}$ & pa & $\mathrm{R}$ \\
\hline L.(N.) digitiformis & $\mathrm{pf}$ & pa & $\mathrm{R}$ \\
\hline L.(N.) duarte & $\mathrm{mf}$ & pa & A \\
\hline L.(N.) cruentata & $\mathrm{mf}$ & $\mathrm{ma}$ & $\mathrm{C}$ \\
\hline L.(N.) spl & $\mathrm{mf}$ & pa & $\mathrm{A}$ \\
\hline L.(N.) $\mathrm{sp} 2$ & $\mathrm{mf}$ & pa & $\mathrm{A}$ \\
\hline L.(L.) $\mathrm{sp} 3$ & $\mathrm{f}$ & pa & $\mathrm{A}$ \\
\hline L.(N.) sp4 & $\mathrm{f}$ & pa & $\mathrm{A}$ \\
\hline L.(N) sp5 & $\mathrm{pf}$ & pa & $\mathrm{R}$ \\
\hline L.(N.) sp6 & $\mathrm{f}$ & pa & $\mathrm{A}$ \\
\hline Gonzaga nigriceps & $\mathrm{pf}$ & pa & $\mathrm{R}$ \\
\hline
\end{tabular}

${ }^{a}$ Frequência de ocorrência; ${ }^{b}$ Abundância relativa; ${ }^{c}$ Classe de Abundância 


\section{Distribuição da Abundância de Chrysopidae com relação à densidade do dossel e as fases lunares.}

Foram coletados 529 indivíduos em dossel menos denso e 887 indivíduos no dossel mais denso (Figura 1). Isso evidencia com diferença significativa pelo teste do quí-quadrado $\mathrm{p}<0,01, \mathrm{X}_{\mathrm{gl}=1}^{2}=90,5$, que a densidade do dossel influencia na captura de crisopídeos florestais, onde quanto mais denso o dossel mais propício de ser obtido um maior número de adultos com armadilha atrativa. Para outras metodologias de coleta, principalmente as de coleta ativa, esse parâmetro deve também ser avaliado. Esse resultado possibilita gerar hipótese de que a atratividade dos crisopídeos em ecossistemas florestais seja maior durante as fases não luminosas da lua, pois as áreas eram adjacentes e não era esperada a diferença significativa na abundância da família Chrysopidae verificada para o dossel mais denso.

Figura 1. Abundância de Chrysopidae relacionado à densidade do dossel vegetal, menos denso e mais denso, na Floresta Nacional de Pacotuba, capturados em armadilha atrativa, iscada com solução de melado de cana-de-açúcar de 2012 a 2013.

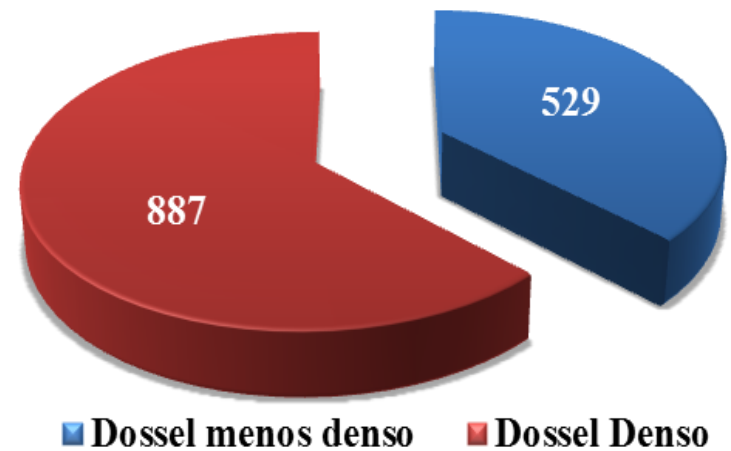

Quando essa avaliação foi realizada avaliando a abundância nas fases da lua, foi possível constatar que na fase luminosa foram capturados 619 contra 797 adultos na fase não luminosa. Essa diferença foi estatisticamente significativa pelo teste do quí-quadrado para $\mathrm{p}<0,01, \mathrm{X}_{\mathrm{gl}=1}^{2} ; 22,4$. (Figura 2).

Figura 2. Abundância de Chrysopidae relacionado às fases luminosas e não luminosas na Floresta Nacional de Pacotuba, capturados em armadilha atrativa, iscada com solução de melado de cana-de-açúcar de 2012 a 2013.

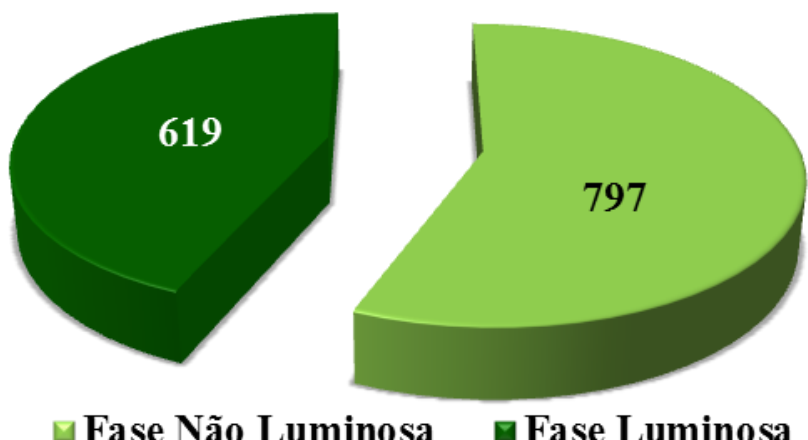

Quando a comparação da abundância de crisopídeos foi referente às fases da lua, foi possível evidenciar que a lua nova apresentou maior registro de indivíduos tanto no dossel mais denso quanto no menos denso (Figura 3 e 4), considerando o pico de captura no período de luminosidade reduzida do intervalo apenas da lua nova que coincidiu como s períodos de captura entre os dias 24 a 26 dos 12 meses 
de avaliação (Figura 5). Esses resultados ainda não foram registrados para outros grupos de insetos, pois é perceptível que alguns pesquisadores como Silva-Filho (2011) e Pontes (2012) trabalharam com cronograma mensal padronizado numa mesma época do mês. Isso possibilitará a captura de maior número de indivíduos.

A espécies $L$. (L.) varia foi captura em todas as fases da lua, mas sua abundância foi maior na lua nova, seguida da fase cheia, crescente e minguante (Tabela 3). Enquanto algumas espécies como $L$. $(L$.) boxi somente foi coletada na sua crescente em ambos os dosséis e G. nigriceps na lua nova, $L$. (N.) digitiformis na lua cheia (Tabela 3 e 4). Esses resultados de ocorrência para essas três últimas espécies não são expressivos para concluir sobre a influência das fases para essas espécies, devido o número de indivíduos capturados ter sido bem reduzido.

Figura 3. Abundância de Chrysopidae relacionado às distintas fases da lua, Minguante - MING; Nova NOVA; Crescente - CRESC; Cheia - CHEIA, referente à menor densidade do dossel vegetal na Floresta Nacional de Pacotuba, capturados em armadilha atrativa, iscada com solução de melado de cana-de-açúcar de 2012 a 2013.

\section{Dossel menos denso}
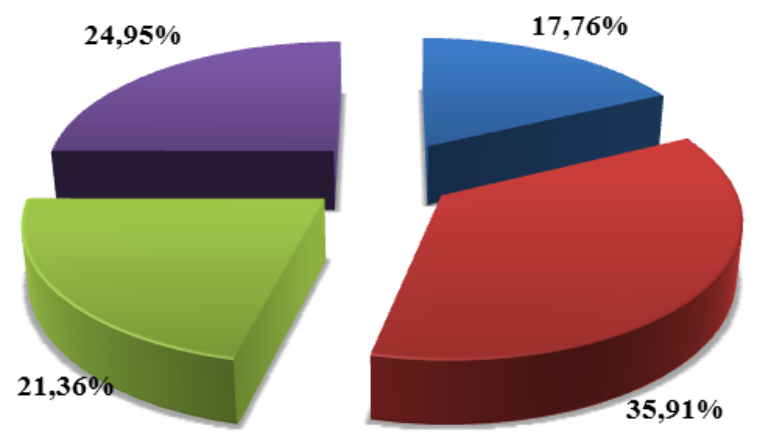

口 $\mathrm{MING}$

NOVA

$\triangle \mathrm{CRESC}$

$\square$ CHEIA

Figura 4. Abundância de Chrysopidae relacionado às distintas fases da lua, Minguante - MING; Nova NOVA; Crescente - CRESC; Cheia - CHEIA, referente à maior densidade do dossel vegetal na Floresta Nacional de Pacotuba, capturados em armadilha atrativa, iscada com solução de melado de cana-de-açúcar de 2012 a 2013.

\section{Dossel mais denso}

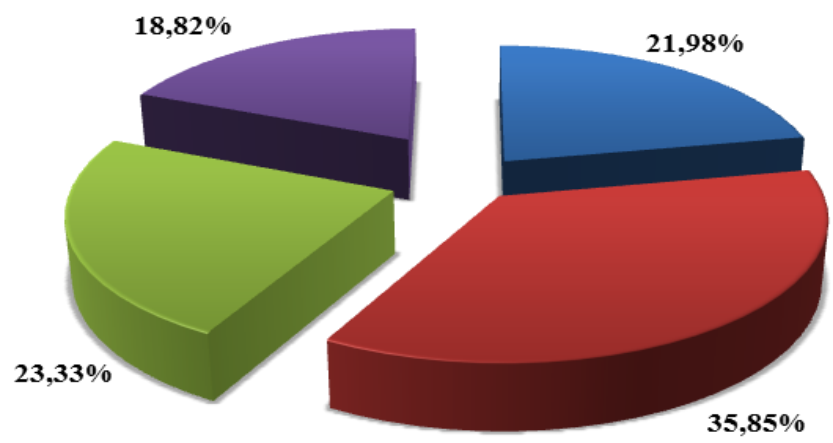

$\square \mathrm{MING}$

ⓃOVA

$\square \mathrm{CRESC}$

$\square$ CHEIA 
Tabela 3. Distribuição da abundância e riqueza de Chrysopidae relacionadas à densidade do dossel vegetal, menos denso e mais denso, e às fases da lua Minguante - MING; Nova - NOVA; Crescente CRESC; Cheia - CHEIA, na Floresta Nacional de Pacotuba, capturados em armadilha atrativa, iscada com solução de melado de cana-de-açúcar de 2012 a 2013.

\begin{tabular}{|c|c|c|c|c|c|c|c|c|c|}
\hline \multirow{3}{*}{ ESPÉCIES } & \multicolumn{4}{|c|}{ Dossel menos denso } & \multicolumn{4}{|c|}{ Dossel mais denso } & \multirow{3}{*}{ TOTAL } \\
\hline & \multicolumn{8}{|c|}{ FASES DA LUA } & \\
\hline & MING & NOVA & $\overline{\text { CRESC }}$ & CHEIA & MING & NOVA & $\overline{\text { CRESC }}$ & CHEIA & \\
\hline L. (L.) varia & 84 & 168 & 103 & 129 & 175 & 286 & 176 & 154 & 1275 \\
\hline L. (L.) boxi & - & - & 1 & - & - & - & 1 & - & 2 \\
\hline L. $(N$.$) digitiformis$ & - & - & - & - & - & - & - & 1 & 1 \\
\hline L. (N.) duarte & 1 & 1 & - & - & 3 & 4 & 10 & 1 & 20 \\
\hline L. $(N$.$) cruentata$ & 4 & 11 & 6 & 2 & 9 & 19 & 16 & 8 & 75 \\
\hline L. (N.) sp1 & 1 & 1 & - & - & 2 & 2 & 1 & 1 & 8 \\
\hline L. (N.) $\mathrm{sp} 2$ & 3 & 7 & 1 & - & 3 & 3 & 1 & 2 & 19 \\
\hline L. (L.) $\mathrm{sp} 3$ & - & 1 & - & - & - & - & 1 & 1 & 3 \\
\hline L. $(N.) \mathrm{sp} 4$ & - & - & 1 & 1 & 1 & - & - & - & 3 \\
\hline L. (N) sp5 & - & - & - & - & 1 & - & - & - & 1 \\
\hline L. (N.) sp6 & 1 & - & 1 & - & 1 & 3 & 1 & - & 7 \\
\hline G. nigriceps & - & 1 & - & - & - & 1 & - & - & 2 \\
\hline Total & 94 & 190 & 113 & 132 & 195 & 318 & 207 & 167 & 1416 \\
\hline
\end{tabular}

Tabela 4: Riqueza e Abundância das fases não luminosa e luminosa, na Floresta Nacional de Pacotuba, capturados em armadilha atrativa, iscada com solução de melado de cana-de-açúcar de 2012 a 2013.

\begin{tabular}{|c|c|c|c|c|}
\hline \multirow{2}{*}{ Espécies } & \multicolumn{2}{|c|}{ Fazes não luminosa } & \multicolumn{2}{|c|}{ Fazes luminosa } \\
\hline & MING & NOVA & CRESC & CHEIA \\
\hline L. (L.) varia & 259 & 454 & 279 & 283 \\
\hline L. (L.) boxi & - & - & 2 & - \\
\hline L. $(N$.$) digitiformis$ & - & - & - & 1 \\
\hline L. (N.) duarte & 4 & 5 & 10 & 1 \\
\hline L. (N.) cruentata & 13 & 30 & 22 & 10 \\
\hline L. $(N.) \mathrm{sp} 1$ & 3 & 3 & 1 & 1 \\
\hline L. $(N.) \mathrm{sp} 2$ & 6 & 10 & 2 & 2 \\
\hline L. (L.) sp3 & - & 1 & 1 & 1 \\
\hline L. $(N.) \mathrm{sp} 4$ & 1 & - & 1 & 1 \\
\hline L. (N) sp5 & 1 & - & - & - \\
\hline L. $(N.) \operatorname{sp} 6$ & 2 & 3 & 2 & - \\
\hline G. nigriceps & - & 2 & - & - \\
\hline Total & 289 & 508 & 320 & 300 \\
\hline
\end{tabular}




\section{Diversidade de Chrysopidae na Floresta Nacional de Pacotuba}

Avaliando a diversidade de Chrysopidae na Flona de Pacotuba foi verificado por intermédio da curva de rarefação associada ao estimador probabilístico de riqueza o Jackniffe 1 que a coleta realizada com a armadilha atrativa foi eficiente na captura de crisopídeos, pois a curva ainda está em ascensão. O estimador do Jack 1 associado a curva também demonstra que às expedições foram suficientes para amostrar as espécies da área. Mesmo com o grande esforço amostral de 40 armadilhas por três dias consecutivos nas quatro fases lunares por 12 meses, o que caracteriza um esforço de 5760 dias de amostragem, a riqueza e abundância foram relativamente pequenas quando comparada aos menores fragmentos amostrados mensalmente por Silva-Filho (2011) na área da Reserva Biológica União no estado do Rio de Janeiro e, Pontes (2012) na área da Reserva de Sooretama no Estado do Espírito Santo.

Figura 5: Curva de rarefação para riqueza em função da abundância de Chrysopidae para o número de expedições, aleatorizadas e simuladas a 1000 vezes, para a Floresta Nacional de Pacotuba de Setembro de 2012 a Agosto de 2013. Associada ao valor probabilístico de coleta de espécies, estimador de Jackniffe 1 para o esforço amostral.

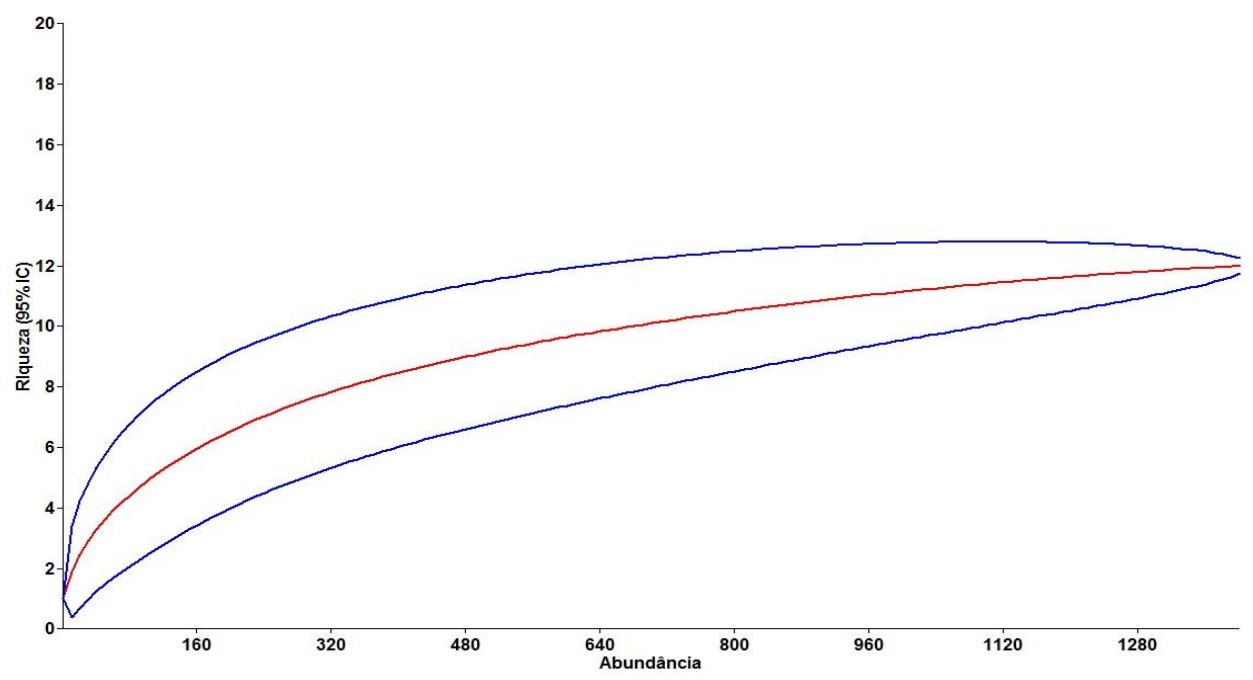

A diversidade de chrysopidae da Floresta Nacional de Pacotuba descrita pelo índice de Shannon foi igual a $\mathrm{H}^{\prime}=0.4442$ (Tabela 5), valor bem inferior ao apresentado por Silva-Filho (2011) para a menor em duas áreas de Floresta Ombrófila Submontana e de Terras Baixas do Rio de Janeiro $\left(H^{\prime}=0,999\right.$ e H'= 1,150). Em relação a outros dois levantamentos envolvendo a taxocenose de Chrysopidae em Floresta Estacional Semidecidual de Minas Gerais, a diversidade em Chrysopidae desse trabalho também foi menor bem menor quando comparado a 1,88 (SOUZA et al, 2008) e 2,02 (COSTA et al., 2010). A diversidade nas comparações com levantamentos da fauna de crisopídeos em distintos ambientes florestais do domínio Mata Atlântica sustentam que Floresta Nacional de Pacotuba tem um índice de biodiversidade muito baixo quando comparados à trabalhos com tamanho do fragmento relativamente similar ao da Flona de Pacotuba.

Os resultados apresentaram uma dominância de 0,9066 mensurada por Berger-Parker e a equabilidade de Pielou igual a 0,1796 (Tabela 5), representada pela abundância de $L$. (L.) varia que apresentou $91 \%$ do total de indivíduos capturados na área. Esses dados são similares aos evidenciado por Silva-Filho (2011) e Pontes (2012) que verificaram maior abundância desta espécie em torno também de 
90\% do total de espécie. A riqueza de Margalef também é relativamente pequena quando comparada aos outros autores.

Tabela 5: Índices de diversidade de espécies para os Chrysopidae da Floresta Nacional de Pacotuba, capturados em armadilha atrativa, iscada com solução de melado de cana-de-açúcar de 2012 a 2013.

\begin{tabular}{cc}
\hline Índices & Valores \\
\hline Taxa_S & 12 \\
Individuals & 1413 \\
Shannon_H & 0.4462 \\
Margalef & 1.517 \\
Equitability_J & 0.1796 \\
Berger-Parker & 0.9066 \\
\hline
\end{tabular}

Influência da temperatura, umidade relativa, precipitação e velocidade do vento na abundância e riqueza de Chrysopidae na Floresta Nacional de Pacotuba.

Avaliando a influência das variáveis abióticas na captura de crisopídeos como forma de avaliar se a abundância e riqueza deste táxon são influenciadas significativamente por essas variáveis, a análise de regressão linear demonstrou relação, mesmo que baixa, significativamente que apenas a precipitação $\left(r^{2}=0,344 ; p=0,035\right)$ e a velocidade do vento $\left(r^{2}=0,325 ; p=0,042\right)$ na abundância de adultos de Chrysopidae (Figura 3). Tauber e Tauber (2010) consideraram que a temperatura precipitação e Umidade são os principais fatores que influenciam na riqueza e abundância de Chrysopidae. A influência da temperatura na captura de Chrysopidae foi também verificada no levantamento deste grupo taxonômico na Reserva Biológica União e Parque estadual do Desengano (SILVA-FIHLO, 2011).

A velocidade do vento pode ter sido significativa devida ao transporte da pluma de odor às distâncias mais longas o que pode ter possibilitado a maior captura de indivíduos. 
Figura 6: Regressões significativas das variáveis climáticas com os parâmetros de abundância e riqueza de Chrysopidae na Floresta Nacional de Pacotuda, período de setembro de 2012 a agosto de 2013.
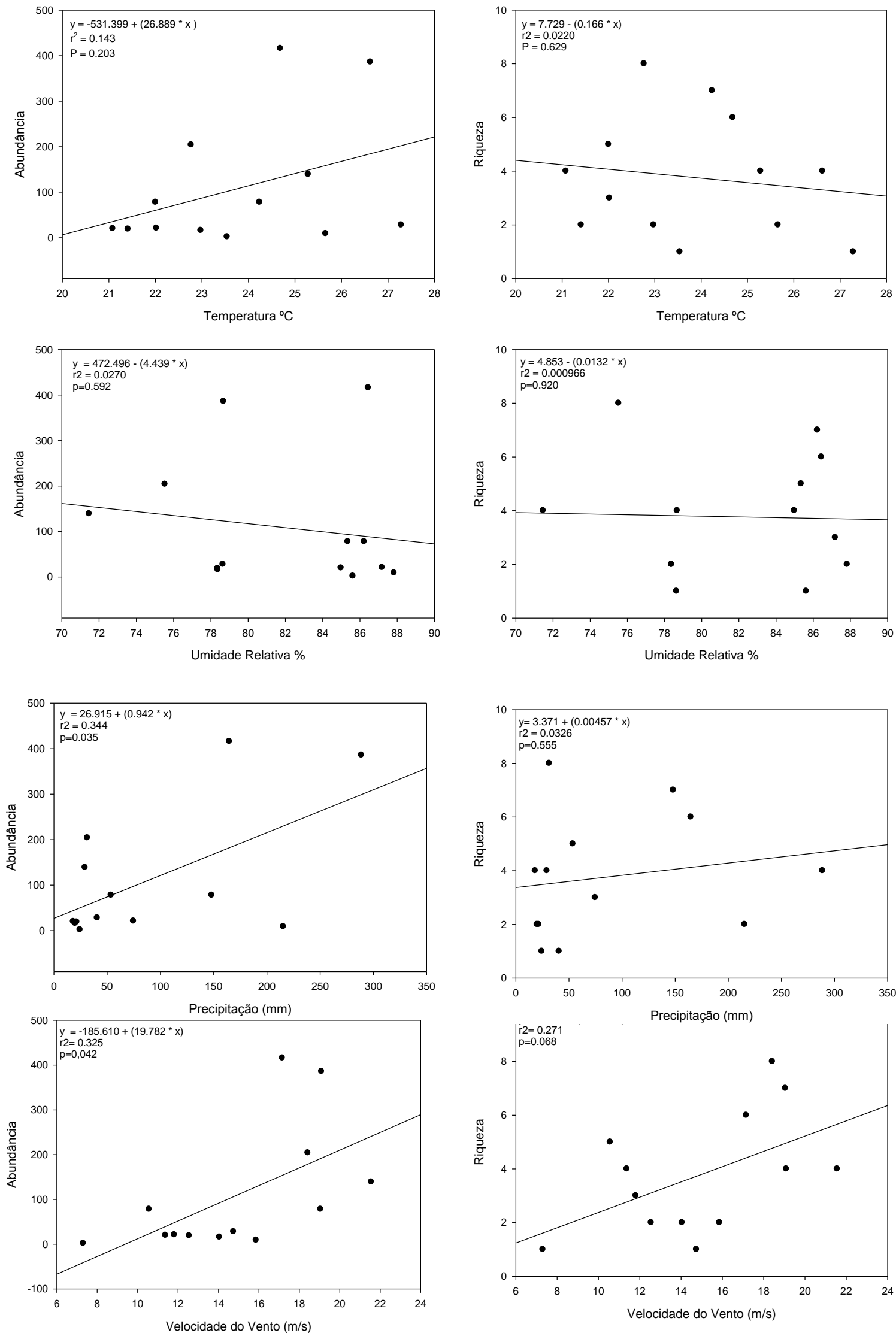


\section{CONCLUSÃO}

A riqueza de Chrysopidae foi relativamente baixa quando comparado com outros trabalhos realizados em ecossistemas florestais com o mesmo grupo taxonômico.

Foi verificado que a abundância de Chrysopidae foi maior quanto mais denso foi o dossel.

Foi evidenciada que a maior abundância deste táxon foi relacionada significativamente às fases escuras da lua, principalmente na lua nova.

A abundância foi influenciada pela precipitação e velocidade do vento, sendo este um fator essencial de difusão dos odores da solução do melado de cana-de-açúcar

\section{AGRADECIMENTOS}

A Fundação de Amparo à Pesquisa e Inovação do Espírito Santo (Fapes) e a Coordenação de Aperfeiçoamento de Pessoal de Nível Superior (Capes).

\section{REFERÊNCIAS}

ALBUQUERQUE, G.S., TAUBER, C.A., TAUBER, M.J. Chrysoperla externa and Ceraeochrysa spp.: potential for biological control in the New World tropics and subtropics. In: McEwen, P.K., New, T.R., Whittington, A.E. (eds.) Lacewings in the crop environment. Cambridge: Cambridge University Press, p. 408-423, 2001.

AYRES, M. et al. Aplicações Estatísticas nas Áreas das Ciências Bio-Médicas. 4. ed. Pará: 2007.

BROOKS, S. J.; BARNARD, P. C. The green lacewings of the world: a generic review (Neuroptera: Chrysopidae). Bulletin of the British Museum of Natural History (Entomology), p. 117-286, 1990.

BUGONI, ALINE. A Importância dos fragmentos florestais na conservação da biodiversidade: O caso dos besouros Escarabeídeos em um fragmento de mata Atlântica próximo de áreas agrícolas. julho, p.1-21, 2012.

BUSCHINI, M.L.T. Species diversity and community structure in trap-nesting bees in Southern Brazil. Apidologie., p. 58-66, 2000.

CARVALHO, C.F., SOUZA, B. Métodos de criação e produção de crisopídeos. In: Bueno, V.H.P. (ed.). Controle biológico de pragas: produção massal econtrole de qualidade. Lavras: UFLA., p. 91-109, 2000.

COSTA, R.I.F., SOUZA, B., FREITAS, S. Dinâmica espaço-temporal de taxocenoses de crisopídeos (Neuroptera: Chrysopidae) em ecossistemas naturais. Neotropical Entomology, 39: 470-475, 2010.

COLWELL, R.K. EstimateS: Statistical estimation of species richness and shared species from samples. Version 8.2.0. User's Guide and application, 2009. Disponível em: http://viceroy.eeb.uconn.edu/ Acesso em 20/08/20015.

COLWELL, R. K.; CODDINGTON, J. A. Estimating terrestrial biodiversity through extrapolation. Philosophical Transactions of the Royal Society of London B., p. 101-118, 1994.

DELFINA, M. C.; TESTON, I. A. Arctiinae(Lepidoptera,Arctiidae) ocorrente na área de pastagem na Amazonia Oriental em Altamira, Pará Brasil. Actta Amazonia.vol.43,n.1., p. 81-89, 2010. 
DÍAZ-ARANDA, L.M. et al. Recognition of early stages of Chrysopidae. In: McEwen, P., New, T., Whittington, A.E. (eds.) Lacewings in the crop environment. Cambridge: Cambridge Univ. Press, p. 6081,2001 .

DUELLI, P. et al. Forest edges are biodiversity hotspots - also for Neuroptera. Acta Zoologica Academiae Scientiarum Hungaricae, p.75-87, 2002.

FREITAS, S., PENNY, N.D.The green lacewings (Neuroptera: Chrysopidae) of Brazilian agroecosystems. Proceedings of the California Academy of Sciences, p. 245-395, 2001.

HAMMER, O. et al. PAST - Paleontological Statistics ver. 1.12. Disponível em: http://www.folk.uio.no/ohammer/past/ acesso em: 18/04/2003

MELO, A. S. O que ganhamos 'confundindo' riqueza de espécies e equabilidade em um índice de diversidade? Biota Neotropica, p.21-27, 2008.

MULTANI, J.S. Diversidade e abundância de crisopídeos (Neuroptera, Chrysopidae) e interações com presas, parasitóides e fatores abióticos em pomares de goiaba em Campos dos Goytacazes, RJ. Tese (Doutorado em Produção Vegetal) - Campos dos Goytacazes - RJ, Universidade Estadual do Norte Fluminense - UENF, 2008.155p.

MAGURRAM, A. E.; RAMNARINE, I.W. Learned mate recognition and reproductive isolation in guppies, Animal Behaviour, p. 1077-1082, 2004.

PENNY, N. D. A guide to the lacewings (Neuroptera) of Costa Rica. Proceedings of the California. Academy of Sciences, p. 161-457, 2001.

PONTES, Thais Bercot. Caracteristica da Taxocenose de Chrysopidae( Insecta, Neuroptera) na Reserva Biológica de Sooretama. Tese (Doutorado em Produção Vegetal) - Campos dos Goytacazes RJ, Universidade Estadual do Norte Fluminense - UENF, 2012, 73p.

SANT’ANA, A. L.; LOZOVEI, A. L. Influência do ciclo lunar na captura de Aedes scapularis (Diptera, Culicidae) na mata Atlântica do Parana. Iheringia Serie Zoologia, p. 175-18, 2001.

SANTOS, A. J. Estimativas de riqueza em espécies. In: Cullen, L., Jr., Rudran, R., Valladares-Padua, C. (eds.) Métodos de estudos em biologia da conservação e manejo da vida silvestre. Curitiba: Editora da UFPR, 2003.19-41p.

SILVA-FILHO, G. 2011. Propriedades das taxocenoses de Chrysopidae (Insecta, Neuroptera) em remanescentes de mata atlântica nas regiões do parque estadual do desengano e da reserva biológica união, rj, e biologia de Leucochrysa (Nodita) paraquaria (Navás), espécie abundante nesse bioma. Tese de Doutorado em Ecologia e Recursos Naturais, UENF, Campos dos Goytacazes-RJ, 2011.117p.

SIVEIRA, NETO et al . Manual de ecologia dos insetos. Piracicaba: Editora Agronômica, Ceres, p. $419,1976$.

SOUZA, B., COSTA, R.I.F., LOUZADA, J.N.C. Influência do tamanho e da forma de fragmentos florestais na composição da taxocenose de crisopídeos (Neuroptera: Chrysopidae). Arquivos do Instituto Biológico, 75: 351-358, 2008.

STATSOFT, Inc. Statistica (data analysis software system), version 7. www.statsoft.coMm/ acesso em: 05/05/2004.

TAUBER, C.A. Generic characteristics of Chrysopodes (Neuroptera: Chrysopidae), with new larval descriptions and a review of species from the United States and Canada. Annals of the Entomological Society of America, p. 472-490, 2003. 
TAUBER, C.A.; LEÓN, T. Systematics of green lacewings (Neuroptera: Chrysopidae): larvae of Ceraeochrysa from Mexico. Annals of the Entomological Society of America, p.197-209, 2001.

TAUBER, C. A., TAUBER, M. J., ALBUQUERQUE, G. S. Plesiochrysa brasiliensis (Neuroptera: Chrysopidae): larval stages, biology, and taxonomic relationships. Annals of the Entomological Society of America, p. 858-865, 2001.

TAUBER, M.J., TAUBER, C.A., DAANE, K.M., HAGEN. K.S. Commercialization of predators: recent lessons from green lacewings (Neuroptera: Chrysopidae: Chrysoperla). American Entomologist, 46: 26$38,2000$.

TOKESHI, M. Species Coexistence: ecological and evolutionary perspective. London: Blackwell Science Ltd. p. 454,1999.

WILLIANS, C. B.; SINGH, B. P. Effect moonlight activity insect. Nature, p. 853, 1951. 